\title{
Normalization of tumour blood vessels improves the delivery of nanomedicines in a size-dependent manner
}

\section{Citation}

Chauhan, Vikash P., Triantafyllos Stylianopoulos, John D. Martin, Zoran Popović, Ou Chen, Walid S. Kamoun, Moungi G. Bawendi, Dai Fukumura, and Rakesh K. Jain. 2012. "Normalization of Tumour Blood Vessels Improves the Delivery of Nanomedicines in a Size-Dependent Manner." Nature Nanotechnology 7 (6) (April 8): 383-388. doi:10.1038/nnano.2012.45.

\section{Published Version}

doi:10.1038/nnano.2012.45

\section{Permanent link}

http://nrs.harvard.edu/urn-3:HUL.InstRepos:37133881

\section{Terms of Use}

This article was downloaded from Harvard University's DASH repository, and is made available under the terms and conditions applicable to Other Posted Material, as set forth at http:// nrs.harvard.edu/urn-3:HUL.InstRepos:dash.current.terms-of-use\#LAA

\section{Share Your Story}

The Harvard community has made this article openly available.

Please share how this access benefits you. Submit a story.

Accessibility 
Nat Nanotechnol. ; 7(6): 383-388. doi:10.1038/nnano.2012.45.

\title{
Normalization of tumour blood vessels improves the delivery of nanomedicines in a size-dependent manner
}

\author{
Vikash P. Chauhan ${ }^{1,2, \neq}$, Triantafyllos Stylianopoulos ${ }^{1,3, \ddagger}$, John D. Martin ${ }^{1,4}$, Zoran \\ Popović ${ }^{5}$, Ou Chen ${ }^{5}$, Walid S. Kamoun ${ }^{1}$, Moungi G. Bawendi ${ }^{5}$, Dai Fukumura ${ }^{1}$, and Rakesh \\ K. Jain ${ }^{1}$ \\ ${ }^{1}$ Edwin L. Steele Laboratory, Department of Radiation Oncology, Massachusetts General \\ Hospital and Harvard Medical School, Boston, Massachusetts, USA. \\ ${ }^{2}$ Harvard School of Engineering and Applied Sciences, Harvard University, Cambridge, \\ Massachusetts, USA. \\ ${ }^{3}$ Department of Mechanical and Manufacturing Engineering, University of Cyprus, Nicosia, \\ Cyprus. \\ ${ }^{4}$ Department of Chemical Engineering, Massachusetts Institute of Technology, Cambridge, \\ Massachusetts, USA. \\ ${ }^{5}$ Department of Chemistry, Massachusetts Institute of Technology, Cambridge, Massachusetts, \\ USA.
}

\section{Abstract}

The blood vessels of cancerous tumours are leaky ${ }^{1-3}$ and poorly organized ${ }^{4-7}$. This can increase the interstitial fluid pressure (IFP) inside tumours and reduce blood supply to them, which impairs drug delivery ${ }^{8-9}$. Anti-angiogenic therapies - which "normalize" the abnormal blood vessels in tumours by making them less leaky - have been shown to improve the delivery and effectiveness of chemotherapeutics with low molecular-weights ${ }^{10}$, but it remains unclear whether normalizing tumour vessels can improve the delivery of nanomedicines. Here we show that repairing the abnormal vessels in mammary tumours, by blocking vascular endothelial growth factor (VEGF) receptor-2, improves the delivery of small nanoparticles ( $12 \mathrm{~nm}$ diameter) while hindering the delivery of large nanoparticles ( $125 \mathrm{~nm}$ diameter). We utilize a mathematical model to show that reducing vessel wall pore sizes through normalization decreases IFP in tumours, allowing small nanoparticles to enter them more rapidly. However, increased steric and hydrodynamic hindrances, also associated with smaller pores, make it more difficult for large nanoparticles to enter tumours. Our results further suggest that smaller $(\sim 12 \mathrm{~nm})$ nanomedicines are ideal for cancer therapy, owing to superior tumour penetration.

\footnotetext{
"Correspondence and requests for materials should be addressed to R.K.J. jain@ steele.mgh.harvard.edu (RKJ). *these authors contributed equally to this work

Author contributions

V.P.C. and R.K.J. conceived and designed the experiments. T.S. and R.K.J. designed and developed the mathematical model and its simulations. V.P.C., J.D.M., and O.C. performed the experiments. T.S. carried out the mathematical model simulations. V.P.C., T.S., J.D.M., and W.S.K. analyzed the data.Z.P., O.C., W.S.K., M.G.B., and D.F. contributed materials/analysis tools. V.P.C., T.S., and R.K.J. co-wrote the paper. All authors discussed the results and commented on the manuscript.

Additional information

Supplementary information accompanies this paper at www.nature.com/naturenanotechnology. Reprints and permission information is available online at http://npg.nature.com/reprintsandpermissions/.
}

Competing financial interests

The other authors declare no competing financial interests. 
Elevated tumour IFP hinders drug delivery by abolishing fluid pressure gradients that produce rapid convective (flow-driven) penetration into tumours ${ }^{11}$. This limits drug penetration across vessel walls into tumours (transvascular) and through tumour tissue (interstitial) to slow diffusion ${ }^{8}$. Anti-angiogenic therapies can repair tumour vessel abnormalities, such as large heterogeneous pores that facilitate leakiness, by inducing vessel maturation ${ }^{12-13}$. This "vascular normalization" reduces IFP to induce convective penetration of molecules up to the size $(\sim 11 \mathrm{~nm})$ of immunoglobulin-G (IgG) (Supplementary Tables 1 and 2$)^{12-14}$. Through normalization, anti-angiogenic therapies seem to benefit patients with colorectal $^{15}$ and brain tumours ${ }^{16-17}$, potentially through improved drug delivery, reduced chemoresistance, and immune reprogramming ${ }^{10}$. Whether normalizing vessels can improve the delivery of nanomedicines - ranging in size from 10-125nm - is not known. These slow-diffusing large therapeutics provide new hope for cancer treatment ${ }^{18-19}$ and would greatly benefit from convective delivery. Unfortunately, increased hydrodynamic and steric hindrance, from smaller vessel pores caused by normalization, may compromise the advantage from enhanced convection.

To determine how vascular normalization affects nanomedicine delivery, we studied whether the anti-VEGF-receptor-2 antibody DC101 modulates nanoparticle penetration rates in orthotopic mammary tumours in vivo. We used intravital multiphoton microscopy and a system of quantum dot-based nanoparticles with tunable size and fluorescence emission wavelength but identical surface chemistry as probes ${ }^{20}$. With these tools, we measured the effects of DC101 on real-time delivery for particles of $12-125 \mathrm{~nm}$ - the size range of approved nanomedicines. Using the resulting data, we applied a novel physiologically-based mathematical model for drug delivery to tumours to determine how anti-angiogenics affect pore size distributions. Furthermore, we utilized this model to study how pore size distributions can be therapeutically modulated to optimize delivery of different sizes of nanomedicines.

We found that a $5 \mathrm{mg} / \mathrm{kg}$ dose of DC101 transiently decreases vessel diameter in orthotopic E0771 tumours - consistent with the structural vascular normalization "window" measured in mice ${ }^{12-13}$ and patients ${ }^{16}$ (Supplementary Fig. 1). We imaged nanoparticle delivery with or without DC101 in orthotopic 4T1 and E0771 mammary tumours (Fig. 1a, Supplementary Fig. 2). We quantified nanoparticle penetration rates as transvascular mass flux per unit vascular surface area and transvascular concentration difference, often termed the effective permeability $^{21}$. Vascular normalization with $10 \mathrm{mg} / \mathrm{kg}$ DC101 led to a 3.1-fold enhancement of transvascular flux in 4T1 tumours for the smallest $(12 \mathrm{~nm})$ nanoparticles, with no improvement in penetration for the larger $(60 \mathrm{~nm}$ and $125 \mathrm{~nm})$ nanoparticles (Fig. 1b). Similarly, 5mg/kg DC101 led to 2.7-fold improvement in transvascular flux in E0771 tumours for the $12 \mathrm{~nm}$ particles with no enhancement for the larger nanoparticles (Fig. 1c). Some individual control tumours exhibited zero or near-zero penetration rates for the larger nanoparticles, with DC101 therapy apparently shutting down large nanoparticle delivery in several tumours (Fig 1b,c). Consistent with the structural normalization "window," we characterized a functional normalization "window" for nanoparticle delivery. During treatment of mice bearing E0771 tumours with $5 \mathrm{mg} / \mathrm{kg}$ DC101 every 3 days, the transvascular flux of $12 \mathrm{~nm}$ particles was enhanced on days 2 and 5 but returned to baseline levels by day 8 (Fig. 2). Importantly, a $10 \mathrm{mg} / \mathrm{kg}$ dose seemed to hinder nanoparticle delivery in E0771 tumours (Supplementary Fig. 3), suggesting a need for judicious dosing based on vascular sensitivity in each tumour and host.

To study how changes in vascular pore size distribution can bring about this complex sizedependent improvement in nanoparticle penetration rates, we developed a mathematical model of drug delivery to tumours (details in the Supplementary Information). The tumour vasculature is represented by a two-dimensional percolation network with one inlet and one 
outlet, which has been shown to resemble the vascular structure and function of tumours (Fig. 3a $)^{6,22}$. It involves a series of interconnected nodes representing vessel segments. Each node is assigned a pore size, assuming a unimodal pore size distribution throughout the tumour vasculature based on previous studies ${ }^{1,23}$. We assume axial Poiseuille-type blood flow $^{24-25}$. Drug exchange with the interstitial space follows Starling's approximation for both diffusive and convective mass flux ${ }^{24}$. Interstitial drug transport also occurs by diffusion and convection, with interstitial fluid flow driving convection calculated using Darcy's law. We use pore theory for the transport of spherical particles through cylindrical pores ${ }^{26-27}$ to calculate the hindrances to diffusion and convection for each pore size ${ }^{24}$. We first solve the steady state fluid problem requiring the net fluid accumulation at each node to be zero and determine the microvascular pressure (MVP) and IFP (Supplementary Figs. 4-6).

Subsequently, we solve the transient drug delivery problem and calculate transvascular flux versus particle size as in the experiment. Model parameters were based on previous studies (Supplementary Tables 3 and 4).

Applying the model, we studied how changes in the mean and heterogeneity (standard deviation) of the vascular pore size distribution affect fluid pressure profiles and drug delivery in tumours. Consistent with previous studies, large heterogeneous pores result in elevated IFP in the centre of the tumour with no transvascular pressure difference, $\Delta \mathrm{P}=$ MVP - IFP (Fig. 3b). Smaller, more homogenous pores produce low IFP with a non-zero $\Delta \mathrm{P}$ - the result of vascular normalization - that induces convection (Fig. 3b). We simulated penetration rates for therapeutics from $1-250 \mathrm{~nm}$ in size while varying mean pore sizes from $40-1000 \mathrm{~nm}$ (diameter $)^{1}$ for homogenous, moderate, or heterogeneous pores corresponding to $20 \mathrm{~nm}, 60 \mathrm{~nm}$, or $100 \mathrm{~nm}$ pore size standard deviations respectively (area fractions held constant; Fig. 3c). Generally, smaller therapeutics $(1-12 \mathrm{~nm})$ demonstrate the most rapid tumour penetration, while the largest therapeutics $(125-250 \mathrm{~nm})$ did not appreciably leave the vasculature (Fig. 3d). Importantly, convection is dominant at small mean pore sizes. Increasing the mean pore size past a point $(>140 \mathrm{~nm})$ hinders nanoscale therapeutic delivery due to rising IFP leading to limited convection, leaving diffusion dominant (Fig. 4a). This effect couples with increasing hydrodynamic and steric hindrance to transport as pore sizes approach therapeutic particle size (Fig. 4b). Therefore, each size of therapeutic has its own ideal mean pore size for maximal delivery to tumours. This ideal mean pore size becomes larger with therapeutic size, though increasing pore size heterogeneity broadens these maxima towards smaller mean pore sizes for therapeutics larger than $12 \mathrm{~nm}$.

To investigate potential translational implications of these findings, we studied whether vascular normalization enhances the effectiveness of anti-cancer nanomedicines. We compared two clinically-used nanomedicines with widely varied sizes - Doxil, with a diameter of $\sim 100 \mathrm{~nm}$, and Abraxane, which attains a size of $\sim 10 \mathrm{~nm}$ upon dilution in plasma (Supplementary Fig. 7). Considering the 5 day normalization window we characterized for nanoparticles, we treated mice bearing orthotopic E0771 mammary tumours with DC101 $(5 \mathrm{mg} / \mathrm{kg})$ or non-specific $\mathrm{IgG}$ on days 0 and 3 while treating with Doxil $(2 \mathrm{mg} / \mathrm{kg})$ or Abraxane (10mg/kg) on days 1-5 (Fig. 5a). We quantified the effect of each combination therapy based on the time to reach double the initial volume (Fig. 5b). While both Doxil and Abraxane monotherapy induced a similar growth delay, vascular normalization with DC101 enhanced the effectiveness of only the $\sim 10 \mathrm{~nm}$ Abraxane while not affecting that of the $\sim 100$ nm Doxil.

Combining our experimental and simulated results, several conclusions can be made. The experiments suggest that vascular normalization with anti-angiogenic therapies will only enhance delivery and effectiveness for relatively small therapeutics - including smallmolecule chemotherapeutics, biologics, and small nanoparticles. Together with the model, these data support the general, yet experimentally unproven, concept that vascular 
normalization reduces vessel pore sizes (Supplementary Fig. 8). This reduces IFP leading to a non-zero $\Delta \mathrm{P}$ throughout tumours, an effect seen experimentally ${ }^{12,15}$, which our model shows can restore convective drug penetration. Unfortunately, smaller and more homogenous pores can also hinder transport for large therapeutics, resulting in diminished or unimproved tumour penetration for larger nanoparticles in both our experiments and simulations. The simulations also predict that remodelling vessels to increase pore sizes would enhance delivery for only the largest $(>125 \mathrm{~nm})$ therapeutics, which are size-excluded by small pores. Perhaps most importantly, they show that tumour penetration rates decrease with increasing nanoparticle size - an effect most pronounced for tumours with smaller vessel pores, as with normalization.

Our findings emphasize the importance of size in nanomedicine design by demonstrating that $12 \mathrm{~nm}$ particles penetrate tumours better than larger particles. Physical principles dictate that both diffusive and convective penetration - transvascular and interstitial - are faster for smaller particles $8,18,26-27$. Importantly, most normal organs feature non-sinusoid continuous epithelium that may be either fenestrated or non-fenestrated with pore cutoff sizes of up to $6-12 \mathrm{~nm}^{28}$, suggesting that $12 \mathrm{~nm}$ particles are the smallest that can take advantage of the enhanced permeability and retention (EPR) effect that leads to favourable toxicity profiles for nanomedicine ${ }^{19}$. Indeed, the smallest probe demonstrating selective delivery - a plurality of the injected dose reaching the tumour - through passive EPR is the $\sim 11 \mathrm{~nm} \mathrm{IgG}^{29}$. While vascular-targeted tumour-penetrating ligands can enhance nanoparticle penetration $^{30}$ and tumour cell-targeting can improve uptake and retention ${ }^{19}$, targeting ligands cannot fully overcome tumour penetration barriers made worse by large size 8 . Considering the superior mass flux into tumours and long circulation times for small nanoparticles, along with the large number of patients receiving normalizing antiangiogenics ${ }^{5}$, small size may represent an important new design constraint for anti-cancer nanomedicine.

\section{Methods}

\section{Tumour models}

Orthotopic mammary tumour models were prepared by implanting a small piece $\left(1 \mathrm{~mm}^{3}\right)$ of viable tumour tissue from a source tumour animal into severe combined immunodeficient (SCID) mice bearing mammary fat pad chambers ${ }^{21}$. The tumours were allowed to grow to $3 \mathrm{~mm}$ in diameter. All animal procedures were carried out following the Public Health Service Policy on Humane Care of Laboratory Animals and approved by the Institutional Animal Care and Use Committee of Massachusetts General Hospital.

\section{Treatment}

Mice were treated with 5 or $10 \mathrm{mg} / \mathrm{kg}$ of DC101 (ImClone Systems), using non-specific rat IgG for control treatments, as a $5 \mathrm{mg} / \mathrm{mL}$ solution by intraperitoneal injection on days 0,3 , and 6. Imaging studies were carried out on days 2, 5, and 8 .

\section{Nanoparticle synthesis}

Nanoparticles were prepared and characterized as described previously ${ }^{20}$.

\section{In vivo imaging}

A mixture of nanoparticles with diameters of $12 \mathrm{~nm}$ (476nm emission), $60 \mathrm{~nm}$ (540nm emission), and $125 \mathrm{~nm}$ (625nm emission) was prepared for intravenous injection. Concentrations were adjusted with in vitro calibration to result in roughly equal photoluminescence intensity for all three nanoparticle samples under 800nm multiphoton excitation. Following retro-orbital injection of $200 \mu \mathrm{L}$ with these concentrations, multiphoton 
imaging was carried out as described previously ${ }^{21}$ on a custom-built multiphoton laserscanning microscope using confocal laser-scanning microscope body (Olympus 300; Optical Analysis Corp.) and a broadband femtosecond laser source (High Performance MaiTai, Spectra-Physics). Image slices were taken at $\sim 60 \mathrm{~mW}$ at sample surface with depths from $0-$ $201 \mu \mathrm{m}$, with $2.76 \mu \mathrm{m}$ steps and $2.76 \times 2.76 \mu \mathrm{m}$ pixels. Mosaic images were taken in raster pattern using a motorized stage (H101, Prior Scientific, Inc.) and customized automation software (LabView, National Instruments). Imaging studies were performed with a 20X magnification, 0.95NA water immersion objective (Olympus XLUMPlanFl, 1-UB965, Optical Analysis).

\section{Image analysis}

Images were analyzed using custom analysis software developed in Matlab (The Mathworks) as described previously ${ }^{21}$. The analysis approach involved 3D vessel tracing to create vessel metrics and a 3D map of voxel intensity versus distance to the nearest vessel over time. Images were also corrected for sample movement over time with 3D image registration. The normalized transvascular flux was calculated using

$\frac{J t}{S_{v}\left(C_{v}-C\right)}=P_{\text {eff }}=\lim _{t \rightarrow 0} \frac{\partial}{\partial t} \frac{\int_{r=R}^{\infty} C(r) r \partial r}{\left(C_{v}-C\right) R}$, where $\mathrm{J}_{\mathrm{t}}$ is the transvascular flux, $\mathrm{S}_{\mathrm{v}}$ is the vessel surface area, $\mathrm{C}_{\mathrm{v}}$ is the concentration of the probe in the vessel, $\mathrm{C}$ is the concentration of the probe immediately extravascular, $\mathrm{P}_{\text {eff }}$ is the effective permeability ${ }^{21}, \mathrm{t}$ is time after the initial image, $r$ is the distance from the vessel central axis, and $R$ is the vessel radius at that point along the vessel. Fluorescence intensities were used as these concentrations. The calculation was made as an average over the entire imaged volume for each tumour.

\section{Model equations}

Details of the model and corresponding equations are provided in the Supplementary Information section.

\section{Tumour growth studies}

E0771 mammary tumours were orthotopically implanted in female SCID mice. The mice were split into treatment groups, time-matched for time after implantation and size-matched for tumour volume at this time $\left(110-111 \mathrm{~mm}^{3}\right)$. The mice were treated at this initial size with $5 \mathrm{mg} / \mathrm{kg}$ DC101 or non-specific IgG on days 0 and 3 by intraperitoneal injection. The mice were simultaneously treated with either $2 \mathrm{mg} / \mathrm{kg}$ Doxil, with a diameter of $\sim 100 \mathrm{~nm}$, or $10 \mathrm{mg} /$ $\mathrm{kg}$ Abraxane, with a diameter of $\sim 10 \mathrm{~nm}$, on days $1-5$ by retro-orbital injection. These relative doses are similar to the relative doses for these two nanomedicines in patients. The primary tumours were then measured every 3 days, beginning on day 0 , using callipers. Tumour growth was quantified using the time for each to reach double its initial volume.

\section{Statistical analysis}

The data are presented as means with standard errors. Groups were compared using a Student's t-test, except for tumour growth data - for which we anticipated a non-normal distribution and used an (exact) Mann-Whitney U-test. In pairwise comparisons of groups in tumour growth experiments, $P$ values were adjusted using Holm's method.

\section{Supplementary Material}

Refer to Web version on PubMed Central for supplementary material. 


\section{Acknowledgments}

We thank Julia Kahn and Sylvie Roberge for technical assistance, James Baish for assistance with the mathematical model, and Marek Ancukiewicz for assistance with statistical analysis. We thank ImClone Systems for generously providing DC101. We gratefully acknowledge support from the National Institutes of Health (P01-CA080124, R01CA126642, R01-CA115767, R01-CA096915, R01-CA085140, R01-CA098706, T32-CA073479), DoD Breast Cancer Research Innovator award (W81XWH-10-1-0016), and an FP7 Marie-Curie IRG grant (PIRG08GA-2010-276894).

R.K.J. receives research support from Dyax, MedImmune, and Roche; is a consultant for Dyax and Noxxon; is on the Scientific Advisory Board for Enlight and SynDevRx; is on the Board of Trustees for H\&Q Capital Management; and is a Co-founder of Xtuit.

\section{References}

1. Hobbs SK. Regulation of transport pathways in tumor vessels: role of tumor type and microenvironment. Proc. Natl. Acad.Sci. USA. 1998; 95:4607-4612. [PubMed: 9539785]

2. Nagy JA, Dvorak AM, Dvorak HF. VEGF-A and the induction of pathological angiogenesis. Annu. Rev. Pathol. 2007; 2:251-275. [PubMed: 18039100]

3. Yuan F, et al. Microvascular permeability and interstitial penetration of sterically stabilized (stealth) liposomes in a human tumor xenograft. Cancer Res. 1994; 54:3352-3356. [PubMed: 8012948]

4. Baish JW, et al. Scaling rules for diffusive drug delivery in tumor and normal tissues. Proc. Natl. Acad.Sci. USA. 2011; 108:1799-1803. [PubMed: 21224417]

5. Carmeliet P, Jain RK. Molecular mechanisms and clinical applications of angiogenesis. Nature. 2011; 473:298-307. [PubMed: 21593862]

6. Gazit Y, Berk DA, Leunig M, Baxter LT, Jain RK. Scale-invariant behavior and vascular network formation in normal and tumor tissue. Phys. Rev. Lett. 1995; 75:2428-2431. [PubMed: 10059301]

7. Nagy JA, Chang SH, Dvorak AM, Dvorak HF. Why are tumour blood vessels abnormal and why is it important to know? Br. J. Cancer. 2009; 100:865-869. [PubMed: 19240721]

8. Chauhan VP, Stylianopoulos T, Boucher Y, Jain RK. Delivery of Molecular and Nanoscale Medicine to Tumors: Transport Barriers and Strategies. Annu. Rev. Chem. Biomol. Eng. 2011; 2:281-298. [PubMed: 22432620]

9. Jain RK. Normalization of tumor vasculature: an emerging concept in antiangiogenic therapy. Science. 2005; 307:58-62. [PubMed: 15637262]

10. Goel S, et al. Normalization of the vasculature for treatment of cancer and other diseases. Physiol. Rev. 2011; 91:1071-1121. [PubMed: 21742796]

11. Jain RK, Baxter LT. Mechanisms of heterogeneous distribution of monoclonal antibodies and other macromolecules in tumors: significance of elevated interstitial pressure. Cancer Res. 1988; 48:7022-7032. [PubMed: 3191477]

12. Tong RT, et al. Vascular normalization by vascular endothelial growth factor receptor 2 blockade induces a pressure gradient across the vasculature and improves drug penetration in tumors. Cancer Res. 2004; 64:3731-3736. [PubMed: 15172975]

13. Winkler F, et al. Kinetics of vascular normalization by VEGFR2 blockade governs brain tumor response to radiation: role of oxygenation, angiopoietin-1, and matrix metalloproteinases. Cancer Cell. 2004; 6:553-563. [PubMed: 15607960]

14. Nakahara T, Norberg SM, Shalinsky DR, Hu-Lowe DD, McDonald DM. Effect of inhibition of vascular endothelial growth factor signaling on distribution of extravasated antibodies in tumors. Cancer Res. 2006; 66:1434-1445. [PubMed: 16452199]

15. Willett CG, et al. Direct evidence that the VEGF-specific antibody bevacizumab has antivascular effects in human rectal cancer. Nature Med. 2004; 10:145-147. [PubMed: 14745444]

16. Batchelor TT, et al. AZD2171, a pan-VEGF receptor tyrosine kinase inhibitor, normalizes tumor vasculature and alleviates edema in glioblastoma patients. Cancer Cell. 2007; 11:83-95. [PubMed: 17222792]

17. Sorensen AG, et al. Increased Survival of Glioblastoma Patients who Respond to Antiangiogenic Therapy with Elevated Blood Perfusion. Cancer Res. 2012; 72:402-407. [PubMed: 22127927] 
18. Jain RK, Stylianopoulos T. Delivering nanomedicine to solid tumors. Nat. Rev. Clin. Oncol. 2010; 7:653-664. [PubMed: 20838415]

19. Peer D, et al. Nanocarriers as an emerging platform for cancer therapy. Nature Nanotechnol. 2007; 2:751-760. [PubMed: 18654426]

20. Popovic Z, et al. A nanoparticle size series for in vivo fluorescence imaging. Angew. Chem. Int. Ed. 2010; 49:8649-8652.

21. Chauhan VP, et al. Fluorescent Nanorods and Nanospheres for Real-Time In Vivo Probing of Nanoparticle Shape-Dependent Tumor Penetration. Angew. Chem. Int. Ed. 2011; 50:1141711420.

22. Gazit Y, et al. Fractal characteristics of tumor vascular architecture during tumor growth and regression. Microcirculation. 1997; 4:395-402. [PubMed: 9431507]

23. Hashizume H, et al. Openings between defective endothelial cells explain tumor vessel leakiness. Am. J. Pathol. 2000; 156:1363-1380. [PubMed: 10751361]

24. Baish JW, Netti PA, Jain RK. Transmural coupling of fluid flow in microcirculatory network and interstitium in tumors. Microvasc. Res. 1997; 53:128-141. [PubMed: 9143544]

25. Pozrikidis C, Farrow DA. A model of fluid flow in solid tumors. Ann. Biomed. Eng. 2003; 31:181-194. [PubMed: 12627826]

26. Bungay PM, Brenner $H$. The motion of a closely-fitting sphere in a fluid-filled tube. Int. J. Multiphase Flow. 1973; 1:25-56.

27. Deen WM. Hindered transport of large molecules in liquid-filled pores. AIChE Journal. 1987; 33:1409-1425.

28. Sarin H. Physiologic upper limits of pore size of different blood capillary types and another perspective on the dual pore theory of microvascular permeability. J. Angiogenes. Res. 2010; 2:14. [PubMed: 20701757]

29. Matsumura Y, Maeda H. A New Concept for Macromolecular Therapeutics in Cancer Chemotherapy: Mechanism of Tumoritropic Accumulation of Proteins and the Antitumor Agent Smancs. Cancer Res. 1986; 46:6387-6392. [PubMed: 2946403]

30. Ruoslahti E, Bhatia SN, Sailor MJ. Targeting of drugs and nanoparticles to tumors. J. Cell Biol. 2010; 188:759-768. [PubMed: 20231381] 

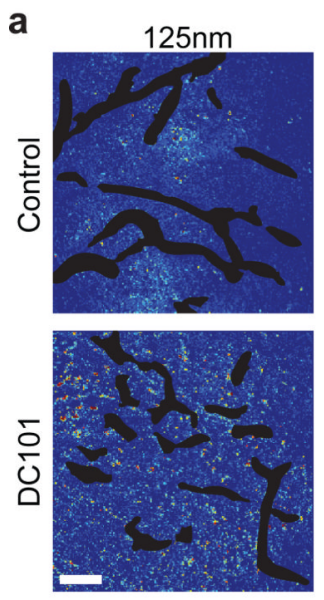
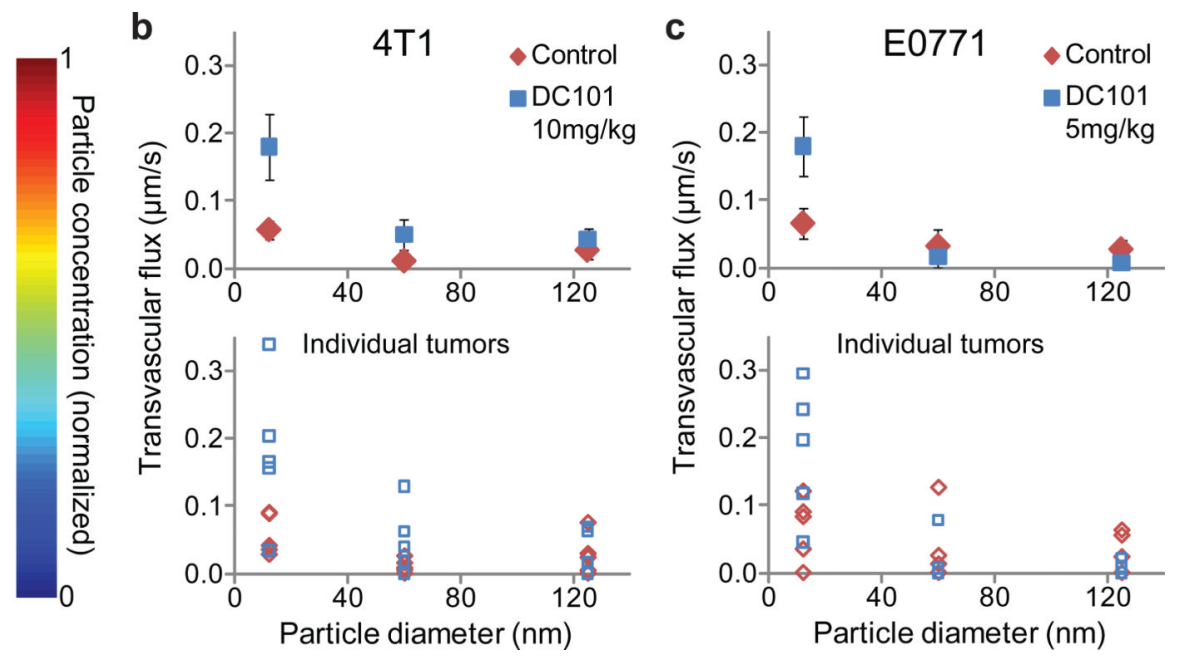

Figure 1. Effects of vascular normalization on nanoparticle delivery in tumours

a, Nanoparticle penetration versus particle size in orthotopic 4T1 mammary tumours in response to normalizing therapy with DC101. Nanoparticle concentrations - denoted by pseudocolour - are relative to initial intravascular levels, with vessels in black.

Normalization improves $12 \mathrm{~nm}$ particle penetration while not affecting $125 \mathrm{~nm}$ penetration. Scale $-100 \mu \mathrm{m}$. b, c, Penetration rates (transvascular flux) for nanoparticles in orthotopic 4T1 and E0771 mammary tumours in mice treated with $10 \mathrm{mg} / \mathrm{kg}$ or $5 \mathrm{mg} / \mathrm{kg}$ DC101, respectively. Closed symbols (top) denote averages by mouse, while open symbols (bottom) are individual tumours. Normalization improves the transvascular flux of $12 \mathrm{~nm}$ particles on day 2 by a factor of 3.1 in 4T1 $(P=0.042$, Student's t-test $)$ and 2.7 in E0771 $(P=0.049$, Student's t-test), while not improving delivery for larger nanoparticles. Normalization also reduces the flux of large nanoparticles to zero in several individual tumours. Animal number $\mathrm{n}=5$ for all groups. 

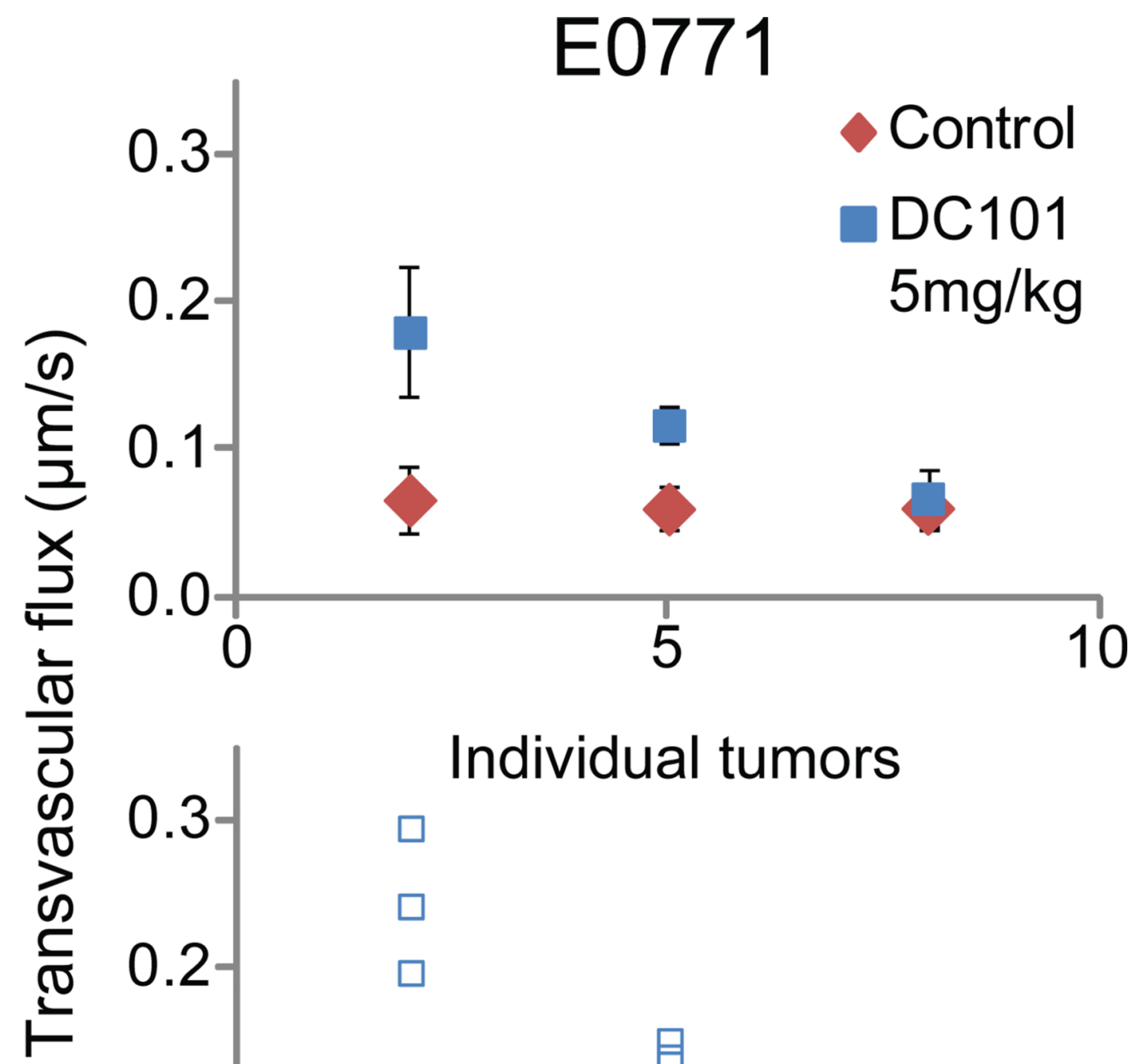

\section{Individual tumors}

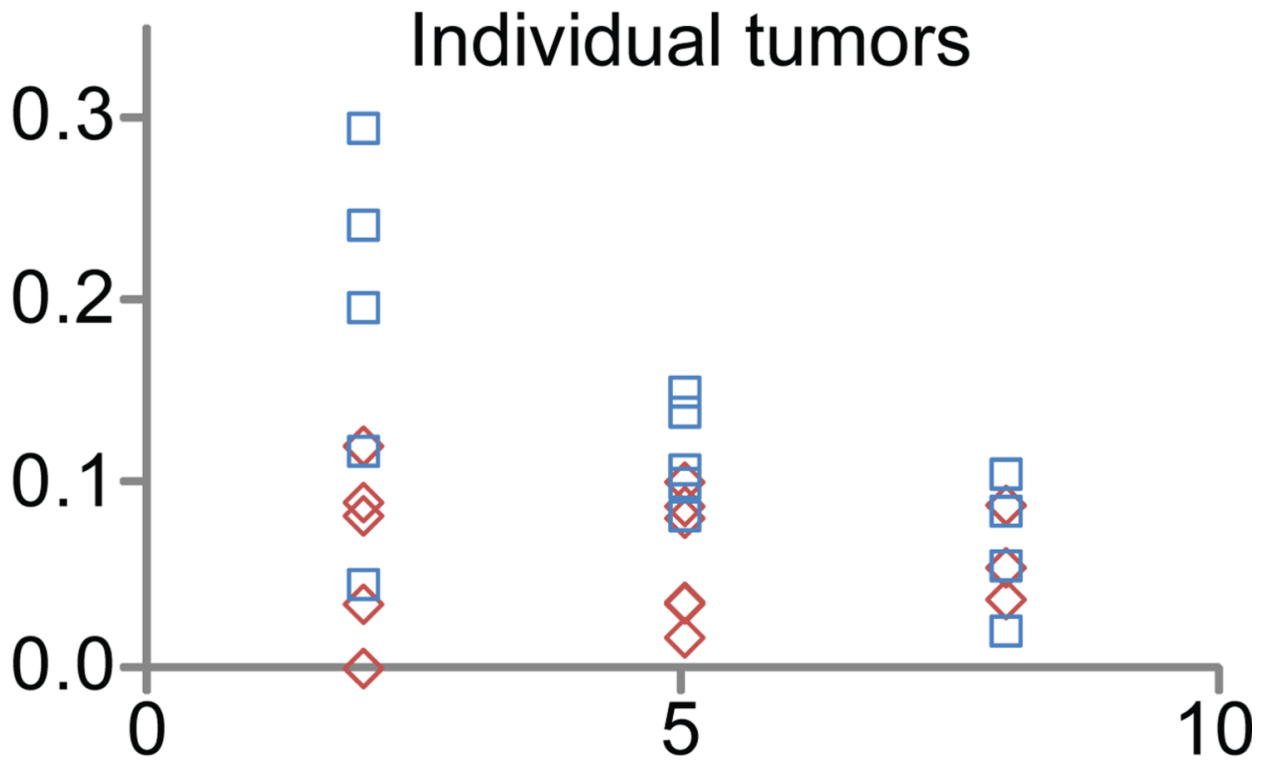

Days of treatment

Figure 2. Functional vascular normalization "window" for nanomedicine delivery Penetration rates (transvascular flux) for 12nm nanoparticles in orthotopic E0771 mammary tumours. Measurements over an 8 day course of treatment with either $5 \mathrm{mg} / \mathrm{kg} \mathrm{DC} 101$ or non-specific rat IgG every 3 days starting on day 0 . Closed symbols (top) denote averages by mouse, while open symbols (bottom) are individual tumours. Treatment with DC101 enhances nanoparticle transvascular flux on days $2(P=0.049$, Student's t-test) and 5 ( $P=$ 0.017 , Student's t-test), with no difference in the treatment groups by day 8 . Animal number $\mathrm{n}=4-5$ for all groups. 
a

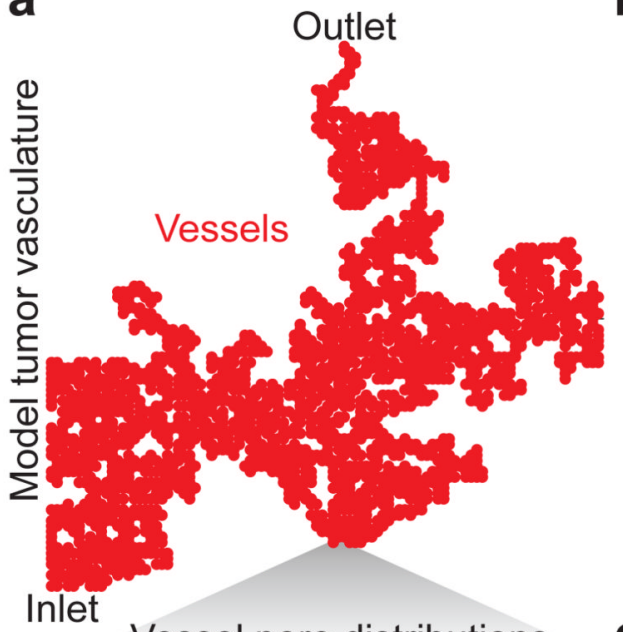

Vessel pore distributions

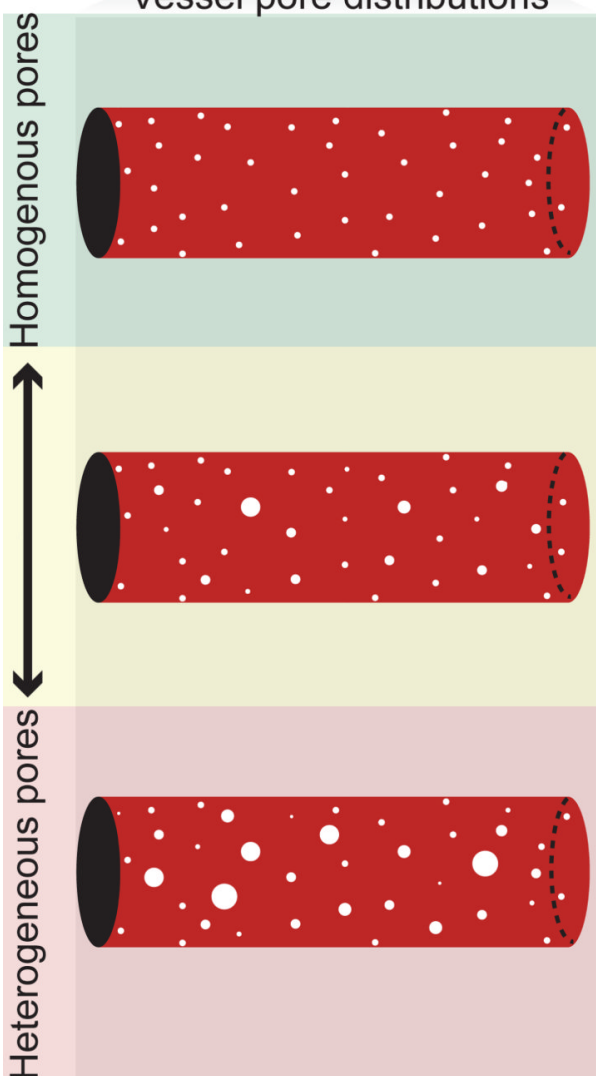

b

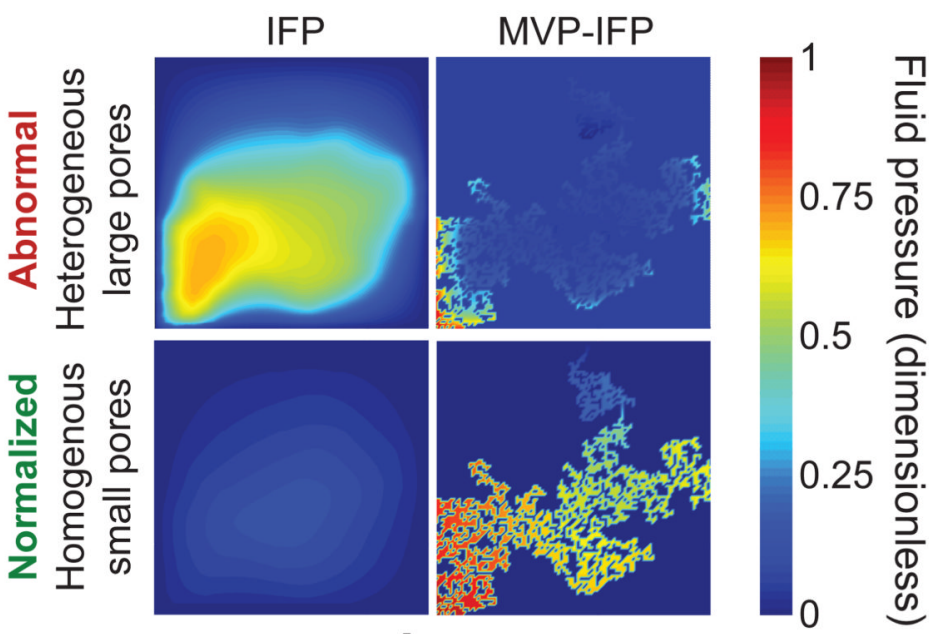

C

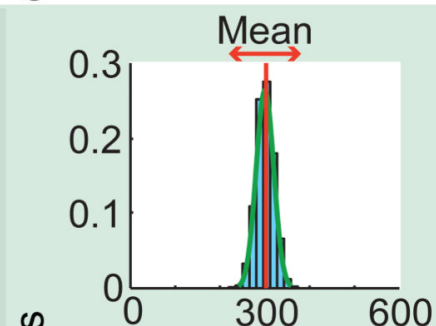

d
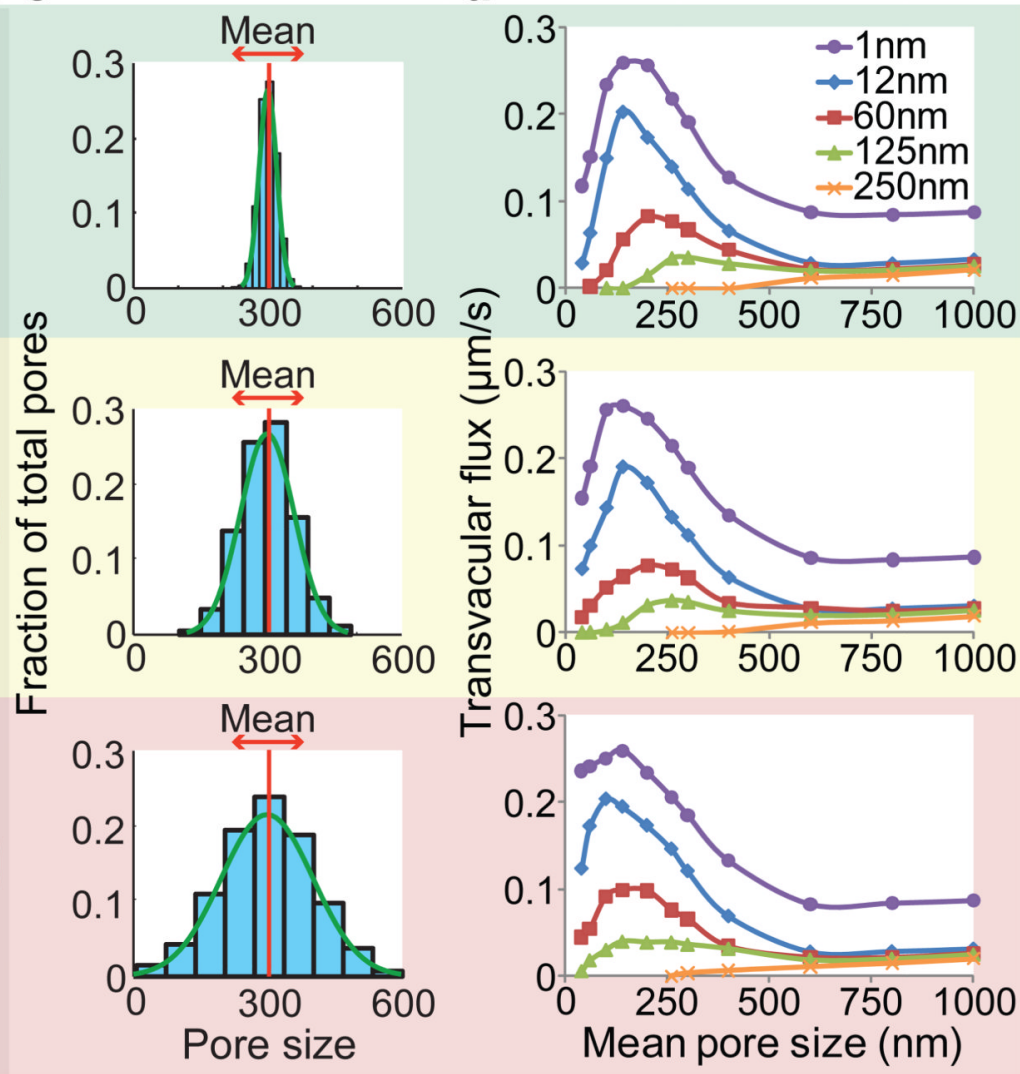

Figure 3. Mathematical model predictions of how changes in vascular pore size distribution affect delivery for different sizes of drugs

a, Model tumour vasculature, formed as a percolation network, with a schematic of vessel pore structure. b. The effect of pore size distribution on fluid pressure. Large heterogeneous pores produce an elevated IFP that approaches the MVP, resulting in a near-zero transvascular pressure gradient (MVP - IFP) for central tumour vessels. Small homogenous pores result in a near-zero IFP and a high transvascular pressure gradient that can drive convective drug delivery. c, The mean pore size (diameter) and pore size standard deviation are varied to predict how pore size changes affect drug delivery. Three standard deviations, at $20 \mathrm{~nm}, 60 \mathrm{~nm}$, or $100 \mathrm{~nm}$, are selected to represent homogenous, moderate, and 
heterogeneous pores respectively. d, Simulations of transvascular flux versus mean pore size and pore size standard deviation for drugs from $1-250 \mathrm{~nm}$ in diameter. 

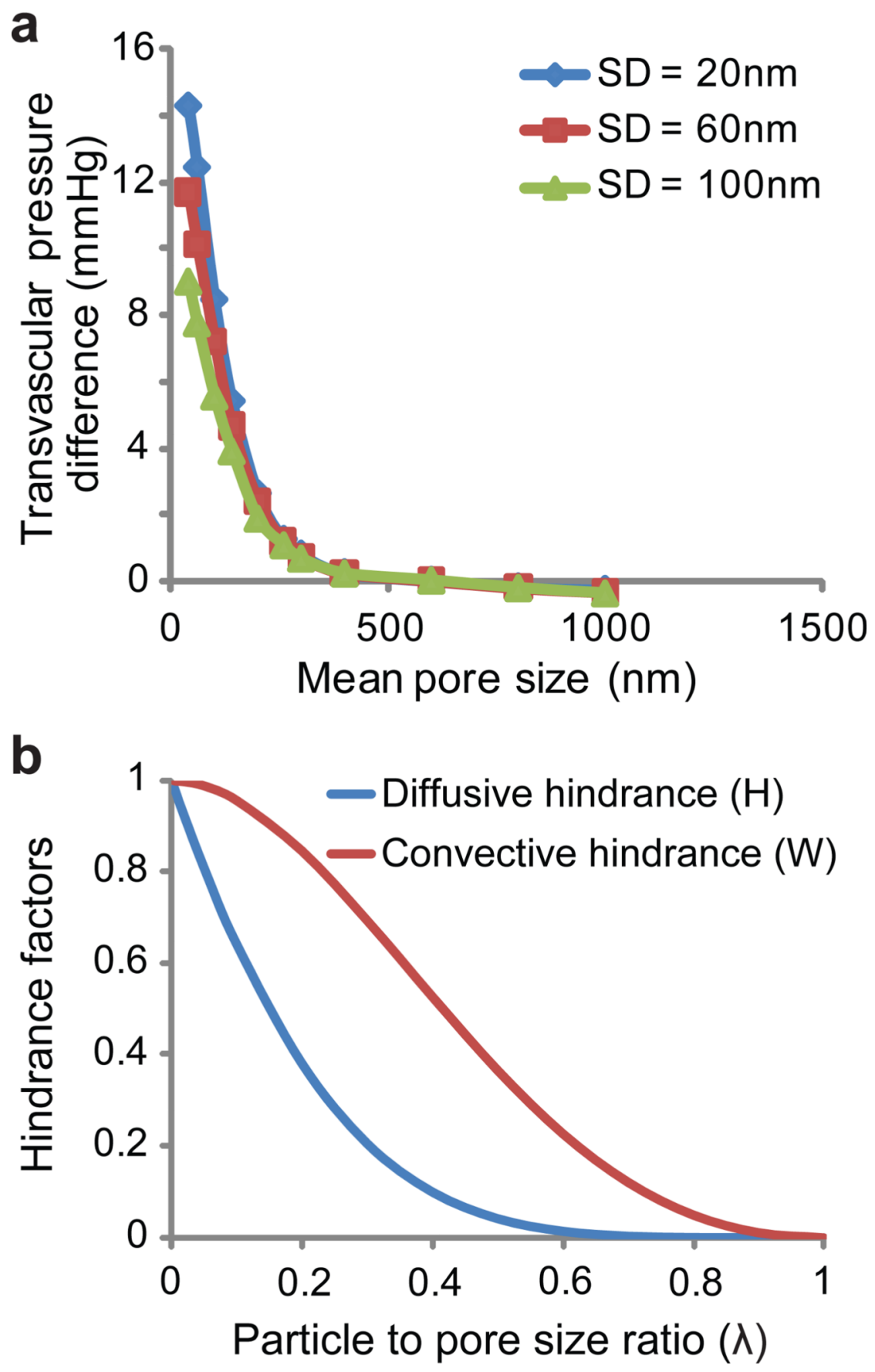

Figure 4. Dependence of the transvascular pressure gradient and transport hindrance on pore size

a, Transvascular pressure difference as a function of the vessel wall pore size. The plot presents the model predictions for three different standard deviations, namely 20,60, and $100 \mathrm{~nm}$. The standard deviation of the distribution affects the transvascular pressure difference only for small pore sizes. For pore size distributions with a mean $>400 \mathrm{~nm}$ the pressure difference, and thus the fluid flux across the vessel wall, is practically zero. b, Hindrance factors for transport through pores versus particle to pore size ratio. The diffusive $(\mathrm{H})$ and convective $(\mathrm{W})$ hindrance factors, which represent hydrodynamic and steric transport hindrance through pores, depend strongly on the particle to pore size ratio $(\lambda)$. A 
hindrance factor of 1 indicates no hindrance, while that of zero denotes no transport whatsoever. Both diffusion and convection are increasingly hindered as particle size approaches pore size. 

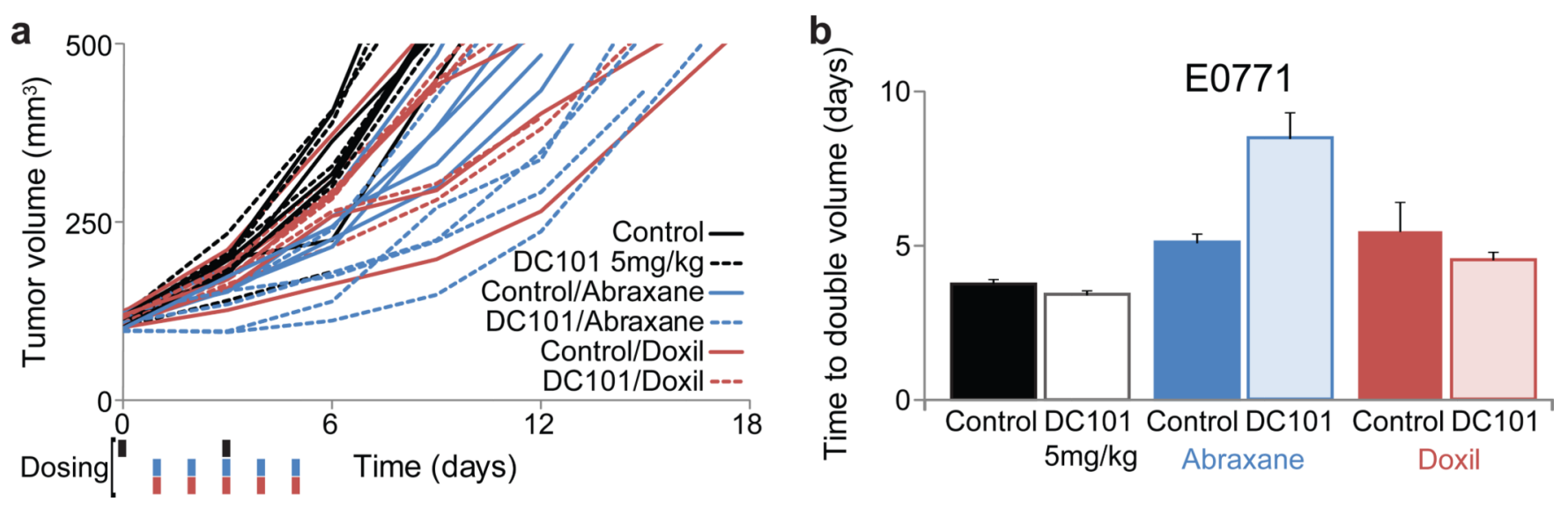

Figure 5. Improvement of cytotoxic nanomedicine effectiveness by vascular normalization a, Volumes of orthotopic E0771 mammary tumours in response to treatment with DC101 or non-specific rat IgG control $(5 \mathrm{mg} / \mathrm{kg}$ on days 0 and 3$)$ in combination with either the $\sim 10 \mathrm{~nm}$ nanomedicine Abraxane (10mg/kg on days 1-5) or the $100 \mathrm{~nm}$ nanomedicine Doxil (2mg/ $\mathrm{kg}$ on days 1-5). b, Quantification of tumour growth rates, based on the time to reach double the initial volume. Abraxane $(P=0.040$, Mann-Whitney U-test $)$ and Doxil $(P=0.040$, Mann-Whitney U-test) monotherapy both induce growth delays versus the control treatment. Normalization with DC101 enhances the effectiveness of the $\sim 10 \mathrm{~nm}$ Abraxane $(P=0.040$, Mann-Whitney U-test), but does not affect that of the $\sim 100 \mathrm{~nm}$ Doxil. Animal number $\mathrm{n}=4-$ 5 for all groups. 